\title{
An unusual cause of empyema in a teenage boy
}

\author{
Jonathan H. Rayment MDCM MSc, Michael Weinstein MD
}

$\mathrm{A}$ previously healthy 17-year-old boy presented to his family physician with an increasingly productive cough and fever of 5 days' duration. Based on a clinical diagnosis of atypical pneumonia, he was prescribed azithromycin. Five days later, his symptoms had not resolved, and left-sided chest pain had developed. His family physician ordered a chest radiograph, which showed consolidation in the lower left lobe. Bacterial pneumonia was diagnosed, and the patient was prescribed high-dose amoxicillin (1g taken orally twice daily).

The patient's symptoms did not improve with amoxicillin therapy, and 2 days after initiation, his cough and chest pain worsened, and he began to experience marked anorexia and malaise. Five days later, he presented to a local hospital with fever, tachypnea and severe respiratory distress. A chest radiograph showed a large left-sided pleural effusion (Figure 1). Although lobar consolidation could not be ruled out based on this chest radiograph, we felt that the radiographic appearance was most likely consistent with reaccumulation of the patient's pleural effusion, especially given his recent history. Broad-spectrum intravenous antibiotics were initiated in the emergency department, and a chest tube was inserted, which drained $400 \mathrm{~mL}$ of turbid fluid. He was transferred to a pediatric hospital with a diagnosis of empyema.

\section{What is the most likely pathogenic agent in this case?}
a. Streptococcus pneumoniae
b. Mycoplasma pneumoniae
c. Influenza A
d. Staphylococcus aureus

The most likely pathogen in this case is $S$. pneumonia (a). Given the unilateral findings on the chest radiograph, $M$. pneumoniae or Influenza A are unlikely to be the cause, because these agents typically result in diffuse, bilateral changes visible on a radiograph. In addition, these agents are infrequently associated with a large parapneu-

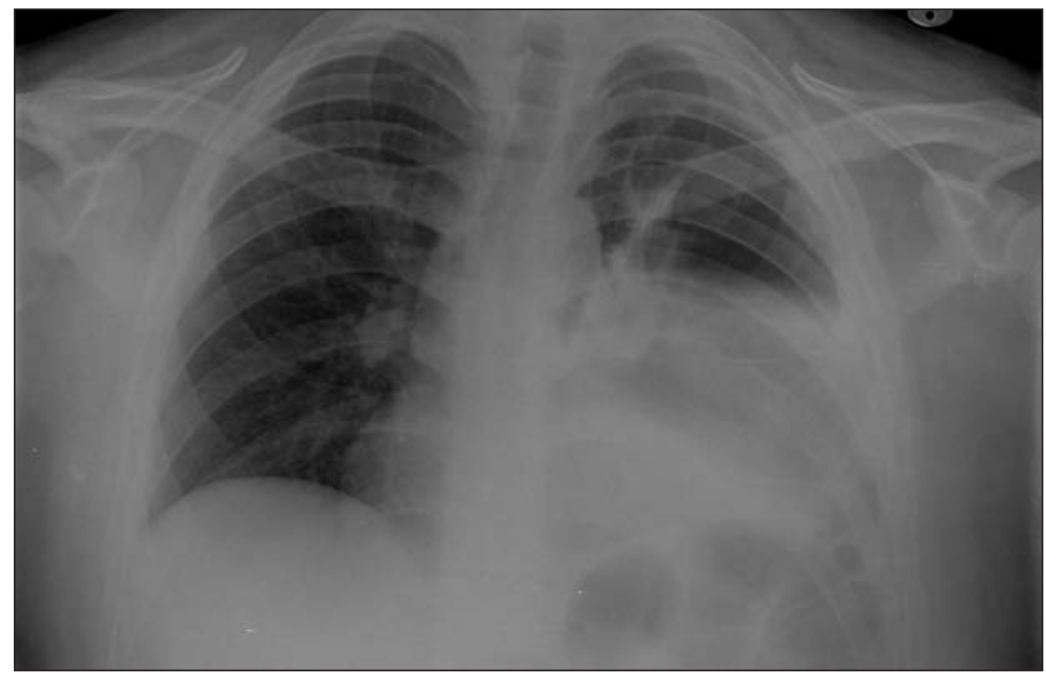

Figure 1: A portable chest radiograph showing a large, left-sided pleural effusion.

monic effusion. Staphylococcus aureus is a less common cause of typical bacterial pneumonia.

Our patient had been previously well, was born in Canada, had no relevant travel history and had no known sick contacts or animal exposures. He did not have epidemiologic risk factors for tuberculosis, and he had received all appropriate vaccinations. He mentioned being briefly submerged in his backyard hot tub 3 weeks earlier. He recalled some coughing at that time, but he did not lose consciousness. Given the history of hot tub submersion, we also considered infection with Pseudomonas aeruginosa.

\section{Which antibiotics would you prescribe?}
a. Ampicillin and gentamicin
b. Meropenem, vancomycin and metronidazole
c. No antibiotics; chest tube drainage is sufficient
d. Cefotaxime, vancomycin and ciprofloxacin

We started intravenous cefotaxime and vancomycin, adding ciprofloxacin for coverage of $P$. aeruginosa (d). Ultrasonography of his chest showed a freeflowing parapneumonic effusion; thus, we decided to proceed with drainage via a chest tube rather than
Competing interests: Michael Weinstein is a member of the board of the Journal of Paediatrics and Child Health. No competing interests declared by Jonathan Rayment.

This article has been peer reviewed.

Correspondence to: Jonathan Rayment, jonathan.rayment@sickkids .ca

CMAJ 2013. DOI:10.1503 /cmaj.122089 
intervene with fibrinolytic or surgical (videoassisted thoracoscopic surgical) therapy.

Aerobic and anaerobic cultures of the blood and pleural fluid showed no growth at 5 days. The results of an acid-fast stain for Mycobacteria, polymerase chain reaction for Mycoplasma, and culture of the pleural fluid for Legionella were negative. A tuberculin skin test was nonreactive. On the eighth day of his stay in hospital, anaerobic Gram-negative bacilli were isolated from the pleural fluid, but these were thought unlikely to be pathogenic.

Over the subsequent 10 days, our patient improved at a rate typical of patients with stage 1 empyema (i.e., exudative). ${ }^{1}$ Intermittent fever persisted up to the time of discharge. Given his clinical condition and laboratory results, intravenous ciprofloxacin was discontinued after a total of 5 days, and vancomycin and cefotaxime were changed to levofloxacin taken orally at 7 days to cover multidrug-resistant $S$. pneumoniae. The patient's chest tube was removed on day 11, and he was discharged home on day 12 with a 4week prescription for oral levofloxacin therapy.

After discharge, high fever and a worsening cough developed. On day 6 after discharge, the patient returned to the pediatric emergency department in substantial respiratory distress. A chest radiograph showed reaccumulation of the leftsided pleural effusion. A chest tube was inserted emergently, and $750 \mathrm{~mL}$ of grossly purulent fluid containing Gram-negative bacilli were drained. Cefotaxime, vancomycin and metronidazole were administered intravenously.

On day 3 of his readmission, anaerobic Gramnegative bacilli were again isolated from the pleural fluid. This isolate and the isolate from the previous admission were analyzed by sequencing of $16 \mathrm{~S}$ ribosomal RNA. In both cases, the results showed sequence homology with Prevotella species.

The patient's clinical status improved rapidly in hospital. His chest tube was removed on day 9 , and he was discharged on day 10 with a 1-month prescription for metronidazole to be taken orally. The patient was well at his 2-week and 1-month follow-up visits, with no signs of recurrence.

\section{Discussion}

In this report, we describe the failed treatment of pneumonia and empyema in a teenage boy who had received timely, guideline-informed empirical treatment. The cause of this treatment failure was Prevotella, an uncommon anaerobic pathogen.

Empyema is defined as the presence of intrapleural pus. The pathophysiology involves several stages. The first stage is a free-flowing parapneumonic effusion (exudative, stage 1). This may progress to a purulent effusion with loculations, which is known as empyema (fibropurulent, stage 2), and, eventually, a fibrinous peel may form (organizational, stage 3 ). ${ }^{2}$

Small pleural effusions can be managed with empiric antibiotic therapy alone., ${ }^{2,3}$ Larger effusions may require surgical intervention as well as antibiotic therapy. In the case of large free-flowing parapneumonic effusions (stage 1), simple percutaneous drainage with an indwelling catheter can, in addition to antibiotic therapy, be sufficient to achieve disease control. ${ }^{2-4}$ In the case of loculated or fibrosed effusions (stage 2 or 3 ), guidelines recommend drainage plus intrapleural fibrinolytics (e.g., tissue plasminogen activator), and management using video-assisted thoracoscopic surgery should be considered. ${ }^{2-4}$ Absolute indications for surgical management are generally thought to be persistent respiratory distress or signs of sepsis despite appropriate antibiotic therapy. ${ }^{2-4}$ Relative indications are less clearly defined, and the most effective intervention modality remains controversial in the literature. ${ }^{2-5}$ The decision to treat should, therefore, take into consideration the patient's clinical status, radiographic findings, local surgical expertise and patient and parent preferences. ${ }^{3,5}$

The empiric selection of antibiotics in the treatment of uncomplicated pneumonia in children is

Table 1: Microbiology, empiric antibiotic therapy and surgical therapy of empyema in children

\begin{tabular}{|c|c|c|}
\hline Bacterial causes of empyema ${ }^{6-8}$ & Empiric antibiotic therapy ${ }^{3,5,9}$ & Surgical therapy ${ }^{*-5}$ \\
\hline $\begin{array}{l}\text { - Streptococcus pneumoniae } \\
\text { - Staphylococcus aureus } \\
\text { - Group A Streptococcus }\end{array}$ & $\begin{array}{l}\text { - Third-generation cephalosporin } \\
\text { (intravenous) }\end{array}$ & \multirow[t]{3}{*}{$\begin{array}{l}\text { - Chest tube drainage, } \\
\text { and/or fibrinolytics } \\
\text { and/or video-assisted thoracic } \\
\text { surgery }\end{array}$} \\
\hline $\begin{array}{l}\text { - Methicillin-resistant S. aureus } \\
\text { (in some geographic regions) }\end{array}$ & $\begin{array}{l}\text { - Third-generation } \\
\text { cephalosporin (intravenous) } \\
\text { plus vancomycin or clindamycin }\end{array}$ & \\
\hline $\begin{array}{l}\text { - Anaerobic bacteria (mostly in } \\
\text { children with impaired airway } \\
\text { defenses) }\end{array}$ & $\begin{array}{l}\text { - Third-generation cephalosporin } \\
\text { (intravenous) plus clindamycin }\end{array}$ & \\
\hline
\end{tabular}


based on decades of observational microbiological studies. ${ }^{6-8}$ The most common pathogen in pneumonia in preschool-age children is $S$. pneumoniae, and the empiric treatment of childhood bacterial pneumonia is high-dose amoxicillin. ${ }^{3.9} \mathrm{In}$ school-age children and adolescents, atypical organisms (M. pneumoniae or Chlamydia pneumoniae) are seen with increasing frequency. As such, older children with clinical signs of atypical pneumonia (indolent presentation, bilateral clinical and radiographic findings) should be given a macrolide,,${ }^{3,9}$ as was done in this patient's case.

Empyema develops in the context of treatment failure of uncomplicated pneumonia. Treatment failure can be related to a delay in initial treatment or the presence of resistant or unusually virulent organisms. The microbiology of empyema mirrors those of childhood uncomplicated pneumonia, with S. pneumoniae, S. aureus and Group A Streptococcus being the most common agents. ${ }^{7.8}$ There is evidence that pneumococcal serotypes not covered by the 7-valent pneumococcal vaccine (especially $1,3,7 \mathrm{~F}$ and 19A) are more likely to cause empyema, which could account for the observed rise in empyema rates over the past decade. ${ }^{10}$

Empiric administration of a third-generation cephalosporin is the first-line antibacterial management of empyema (Table 1). ${ }^{2-9}$ Vancomycin or clindamycin should be considered in regions where methicillin-resistant $S$. aureus is prevalent., Cefuroxime axetil or amoxicillin-clavulanate are suggested in national guidelines and position statements as oral stepdown antibiotic agents. ${ }^{3.5}$ Levofloxacin is also commonly used for older teenagers and adults in the stepdown management of suspected multidrug-resistant S. pneumoniae disease. ${ }^{11}$ The total duration of antibiotic therapy required in cases of empyema is not known, but most experts recommend at least 4 weeks. ${ }^{3,9}$

This case demonstrates that the presence of an unusual pathogenic organism can lead to treatment failure of uncomplicated pneumonia, even when guidelines are followed (Table 2) ${ }^{1-5}$ Anaerobic organisms are very unusual causes of pneumonia and empyema in neurologically normal, otherwise healthy children. ${ }^{6-8}$ Those with impaired mechanical airway defenses (neurologic injury or tracheostomy) or those who have overgrowth of their normal oral flora are at a higher risk of anaerobic pleuropulmonary infections. Clindamycin, metronidazole, aminoglycosides or quinolones are the agents of choice for expanded coverage for cases in which anaerobic agents are suspected. ${ }^{12}$

\section{Conclusion}

This case underscores the need for clinicians to maintain a high index of suspicion for unusual
Table 2: Causes, interventions and signs of treatment failure in empyema

\begin{tabular}{|ll}
\hline Cause & \multicolumn{1}{c}{ Intervention $^{2-5}$} \\
\hline - Resistant or atypical organism & $\begin{array}{l}\text { Augmentation of antibiotic } \\
\text { therapy }\end{array}$ \\
$\begin{array}{ll}\text { - Complicated parapneumonic } \\
\text { effusion }\end{array}$ & $\begin{array}{l}\text { Fibrinolytics and/or video-assisted } \\
\text { thoracic surgery }\end{array}$ \\
- Necrotizing pneumonia & $\begin{array}{l}\text { Computed tomography and } \\
\text { surgical consultation }\end{array}$ \\
\hline
\end{tabular}

Signs of treatment failure

- Persistent high fever* and other signs of sepsis

- Persistent respiratory distress

- Persistent chest pain

*Intermittent low-grade fevers are expected during the normal course of recovery from empyema and do not necessarily indicate treatment failure. ${ }^{1,5}$

causes of pneumonia and empyema, particularly when the course of illness is protracted and the response to standard treatment is atypical.

\section{References}

1. Cohen E, Mahant S, Dell SD, et al. The long-term outcomes of pediatric pleural empyema: a prospective study. Arch Pediatr Adolesc Med 2012;166:999-1004.

2. Balfour-Lynn IM, Abrahamson E, Cohen G, et al. BTS guidelines for the management of pleural infection in children. Thorax 2005;60(Suppl 1):i1-21.

3. Bradley JS, Byington JS, Shah SS, et al. The management of community-acquired pneumonia in infants and children older than 3 months of age: clinical practice guidelines by the Pediatric Infectious Diseases Society and the Infectious Diseases Society of America. Clin Infect Dis 2011;53:e25-76.

4. Islam S, Calkins CM, Goldin AB, et al. The diagnosis and management of empyema in children: a comprehensive review from the APSA Outcomes and Clinical Trials Committee. J Pediatr Surg 2012;47:2101-10.

5. Chibuk T, Cohen E, Robinson J, et al. Paediatric complicated pneumonia: diagnosis and management of empyema. Paediatr Child Health 2011;16:425-9.

6. Michelow IC, Olson K, Lozano J, et al. Epidemiology and clinical characteristics of community-acquired pneumonia in hospitalized children. Pediatrics 2004;113:701-7.

7. Strachan RE, Cornelious A, Gilbert GL, et al. Bacterial causes of empyema in children, Australia, 2007-2009. Emerg Infect Dis 2011;17:1839-45.

8. Eastham KM, Freeman R, Kearns AM, et al. Clinical features, aetiology and outcome of empyema in children in the north east of England. Thorax 2004;59:522-5.

9. Le Saux N, Robinson J. Pneumonia in healthy Canadian children and youth: practice points for management. Paediatr Child Health (Oxford) 2011;16:417-24.

10. Li ST, Tancredi DJ. Empyema hospitalizations increased in US children despite pneumococcal conjugate vaccine. Pediatrics 2010;125:26-33.

11. Heffelfinger JD, Dowell SF, Jorgensen JH, et al. Management of community-acquired pneumonia in the era of pneumococcal resistance: a report from the drug-resistant Streptococcus pneumoniae therapeutic working group. Arch Intern Med 2000;160:1399-408.

12. Brook I. Anaerobic pulmonary infections in children. Pediatr Emerg Care 2004;20:636-40.

Affiliation: The Department of Paediatrics, the Hospital for Sick Children, Toronto, Ont.

Contributors: Both authors contributed substantially to conception and design of the article and to the interpretation of the data. Jonathan Rayment acquired the data and drafted the article, which was revised by both authors. Both authors approved the final version submitted for publication. 